\title{
Characterization of a two-channel, high resolution hard x-ray microscope using Fresnel zone plates for laser-plasma interaction experiments
}

\author{
A. Do, ${ }^{1, a), b)}$ M. Briat, ${ }^{2}$ A. Chaleil, ${ }^{2}$ C. Rubbelynck, ${ }^{2}$ M. Lebugle, ${ }^{3}$ C. David, ${ }^{3}$ and P. Troussel ${ }^{2}$ \\ ${ }^{1}$ Laboratoire pour l'Utilisation des Lasers Intenses (LULI)—CEA, Polytechnique-X, \\ Université Pierre et Marie Curie (UPMC), Paris VI, CNRS: UMR7605, LULI, École Polytechnique, \\ Route de Saclay, 91128 Palaiseau Cedex, France and LULI, Université Pierre et Marie Curie (Paris 6), \\ 3 Rue Galilée, 9420 Ivry-sur-Seine, France \\ ${ }^{2}$ Commissariat à l'Energie Atomique et aux Energies Alternatives (CEA)—DAM-DIF, Bruyère le Châtel, \\ F-91297 Arpajon, France \\ ${ }^{3}$ Paul Scherrer Institut, CH 5232 Villigen-PSI, Switzerland
}

(Presented 16 April 2018; received 7 May 2018; accepted 18 June 2018; published online 15 October 2018)

\begin{abstract}
New X-ray imaging techniques are currently being developed at the "Commissariat à l'énergie atomique et aux énergies alternatives" in the context of Inertial Confinement Fusion. Fresnel zone plates (FZPs) are being considered as they can perform high-resolution and high-flux imaging in the X-ray domain. Here we present the characterization of a bi-lens FZPs resolution used in an imager prototype, designed for the LULI2000 laser facility. Characterization was performed on a synchrotron radiation facility and on a femtosecond laser facility. The resolution of the two FZP channels was measured to be between $2.4 \mu \mathrm{m}$ and $5.2 \mu \mathrm{m}$ and the expected total resolution for the diagnostic was to be $3.3 \mu \mathrm{m}$. Published by AIP Publishing. https://doi.org/10.1063/1.5039326
\end{abstract}

\section{INTRODUCTION}

One of the limiting factors to achieving fusion ignition are the hydrodynamic instabilities. ${ }^{1}$ Over the last few years, the "Commissariat à l'énergie atomique et aux énergies alternatives" (CEA) has been investigating Fresnel zone plates (FZPs) as imaging optics to be used on a high-resolution imaging microscope for the Laser MegaJoule (LMJ) in the context of Inertial Confinement Fusion (ICF). Other solutions such as Kirkpatrick-Baez ${ }^{2}$ or Wolter-type ${ }^{3}$ microscopes have been preferred so far.

A feasibility study has led to the design of a one channel microscope (FUHRI) using a FZP and a multilayer mirror deployed on the LULI2000 facility. ${ }^{4,5}$ This was later upgraded to have 2 channels (FUHRIx2). ${ }^{13}$ The updated version is composed of two FZPs fabricated on the same chip (called a bi-lens) able to image simultaneously two different K-shell emission lines of $\mathrm{Al}$.

One of the most overlooked metrology parameters of the diagnostic is its spatial resolution. It is very hard to predict the resolution of a FUHRI channel on a different facility, meaning that actual experimental measurements must be obtained. Previous experiments with FZPs ${ }^{2}$ showed that we could estimate the diagnostic experimental resolution $\left(r_{\text {exp }}\right)$ as being bounded between the "monochromatic" resolution $\left(r_{m c h r}\right)$ and the "wide bandwidth" spectrum $\left(r_{w b}\right)$ giving us a first approximation,

$$
r_{m c h r} \leq r_{\exp } \leq r_{w b}
$$

Note: Paper published as part of the Proceedings of the 22nd Topical Conference on High-Temperature Plasma Diagnostics, San Diego, California, April 2018.

a) Author to whom correspondence should be addressed: do6@llnl.gov.

b) Current address: Lawrence Livermore National Laboratory, Livermore, CA, USA. $r_{m c h r}$ was measured on a synchrotron radiation facility (FCM beamline, PTB, Bessy II, Germany $)^{4,5}$ with the help of a knife edge method, whereas $r_{w b}$ was obtained by radiography of a gold grid on a femtosecond laser facility (EQUINOX, CEA, France).

If the diagnostic detector resolution $\left(r_{\text {detector }}\right)$ and the source spectrum are known, it is possible to infer a more precise value for the diagnostic resolution $\left(r_{d i a}\right)$ using the quadratic mean formula,

$$
r_{\text {dia }}^{2}=r_{\text {exp }}^{2}+r_{\text {detector }}^{2} .
$$

\section{FZPS CHARACTERISTICS}

One of the issues that can be encountered while using FZPs is the background created by the 0th diffraction order. In order to remove the 0th order signal from the 1st order image plane, a blocker can be placed at the center of the FZP. For a given diagnostic magnification of $M$, a source diameter of $S$ and a blocker diameter of $D_{b}$ on the detector the 1st order image would have a size of $D_{i 1}=M^{*} S$ and the blocker shadow of approximately $D_{i b}=M^{*} D_{b}$. It is desirable to have $D_{i b} \geq D_{i 1}$ which gives the following condition for the 0th order blocker: $D_{b} \geq S$. Thus, FZPs with diameters higher than $1 \mathrm{~mm}$ will be needed for use on fusion experiments, where the source is typically on the order of a few mm.

In collaboration with the Paul Scherrer Institute (PSI, Switzerland), CEA has designed new gold FZPs with diameters larger than $1 \mathrm{~mm}$ whose characteristics are given in Table I. The FZP labeled $a$ has been designed to work at $1850 \mathrm{eV}$, and the one labeled $b$ at $2050 \mathrm{eV}$ corresponding to the $\mathrm{He}_{\beta}$ and $\mathrm{Ly}_{\beta}$ lines of $\mathrm{Al}$, respectively. The FZPs' diameters are inversely proportional to the last zone width and thus the FZP resolution. This relationship is convenient as we are looking 
TABLE I. FZPs' characteristics.

\begin{tabular}{lccccc}
\hline \hline FZP & Diameter $(\mu \mathrm{m})$ & Last zone width $(\mathrm{nm})$ & Stop diameter $(\mu \mathrm{m})$ & Zone thickness & Stop thickness \\
\hline$a$ & 1396.2 & 120 & 465.4 & $460 \mathrm{~nm} \pm 10 \%$ & $2080 \mathrm{~nm} \pm 10 \%$ \\
$b$ & 1260.0 & & 420 & & \\
\hline \hline
\end{tabular}

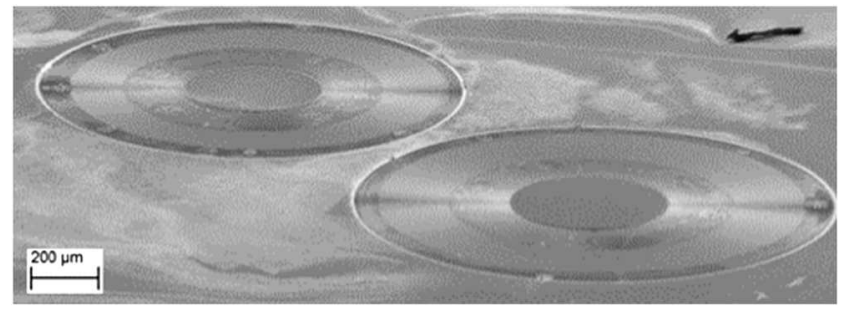

FIG. 1. SEM image of both FZPs.

for high resolution and high diameter, but it also presents a manufacturing challenge as it increases the aspect ratio of the nanostructures. The diameters of the two FZPs were chosen so that they would have the same resolution. Detector constraints imposed a maximum of $1.6 \mathrm{~mm}$ on the centerto-center distance between the FZPs in order to have both images on the same chip. They were placed on a diagonal of a square $\mathrm{Si}$ substrate with a center-to-center distance of $1.4 \mathrm{~mm}$ (Fig. 1).

\section{FZP FABRICATION PROCESS}

The FZPs were manufactured using $100-\mathrm{keV}$ electronbeam lithography (Vistec EBPG 5000plus). A 550-nm thick polymethylmethacrylate (PMMA) resist layer was first patterned with alignment markers on a $275 \mathrm{~nm} \mathrm{Si}_{3} \mathrm{~N}_{4}$ membrane window with a size of $4.5 \mathrm{~mm} \times 4.5 \mathrm{~mm}$. After polymer development, Au was electroplated in the opened trenches using a conductive Au seed layer, ${ }^{6}$ until the desired height of $460 \mathrm{~nm} \pm$ $10 \%$ was reached for optimization of the 1 st order diffraction efficiency of the FZPs. A second electron-beam lithography step was used to produce the central blocker by applying an additional $2800 \mathrm{~nm}$ resist layer, using the previously applied alignment features for alignment to the FZPs. An Au central stop with a thickness of $2080 \mathrm{~nm} \pm 10 \%$ was obtained on the FZPs.

In the context of optical diagnostics of high-temperature plasma at X-ray energies, FZPs offer several important advantages. Such optical elements provide stigmatic imaging, greatly simplifying the design when compared to similar schemes using, e.g., reflective optics pairs. Furthermore, they are largely insensitive to angular alignment and stability, which facilitates their use in complex setups. Finally, the flexibility of the electron-beam lithography approach for patterning makes it easy to change the specifications of the optical system and different designs can be rapidly prototyped. Off-axis imaging FZPs could also potentially be implemented in this context-such a scheme would intrinsically suppress the 0th order radiation, thereby greatly improving the signal to noise ratio of the detected signal.

\section{RESOLUTION MEASUREMENTS}

\section{A. Experiments on BESSY II}

Resolution measurements of the FZPs were performed at the Four-Crystal Monochromator (FCM) beamline in the Physikalisch-Technische Bundesanstalt (PTB) laboratory at the synchrotron radiation facility Bessy II. ${ }^{7}$ The accessible photon energy range of this beamline extends from $1.75 \mathrm{keV}$ to $11 \mathrm{keV}$ using either $\mathrm{Si}$ or InSb crystals as a monochromator. ${ }^{8}$

We used a knife edge method to measure the resolution: The incident synchrotron beam is defined by a square aperture, each side $1200 \mu \mathrm{m}$ long. We use our FZPs to create a focused image of this beam. A photodiode (with active area of $0.5 \mathrm{~cm}^{2}$ ) is placed at a certain distance from the FZP and a sharp edge is moved across the edge of the image. As the sharp edge moves across the beam, we define the Full Width at Half Maximum (FWHM) of the photodiode signal as the resolution of the FZP.

For both FZP $a$ and $b$, we did two sets of horizontal resolution measurements:

- Resolution vs FZP-diode distance for a beam photon energy of $1850 \mathrm{eV}$ (FZP $a$ ) or $2050 \mathrm{eV}$ (FZP $b$ );

- Resolution vs beam photon energy for a FZP-diode distance of $253 \pm 1 \mathrm{~mm}$.

These measurements using a monochromatic source give us a best-case resolution limit for each of the FUHRIx 2 channels. Measurements for both FZPs, presented in Fig. 2, show that their performances are very similar in both cases as expected. Here, for a FZP-diode distance of $253 \mathrm{~mm}$, we obtain a best horizontal spatial resolution of $2.4 \mu \mathrm{m}$. The theoretical resolution of the FZP can be calculated with the formula: ${ }^{9} \sigma_{t h}$ $\approx 1.22^{*} \Delta r_{N} \approx$ few hundreds of $\mathrm{nm}$, with $\Delta r_{N}$ defined as the last zone width of the FZP. The discrepancy comes from the spatial coherence of the synchrotron beam and the quality of the mechanical components (knife edge sharpness and motor step size). When fully illuminated by coherent flux, it was shown previously that diffraction-limited performances can be reached with FZPs, offering sub-micrometric resolution. ${ }^{10}$ Nonetheless, we were able to confirm that the resolution stays stable in a region located around $\pm 1 \mathrm{~mm}$ of the best resolution position; i.e., this is the depth of field. The best focal position of each individual FZP on the chip is different by $0.5 \mathrm{~mm}$, but this is not a problem as this difference is lower than the $1 \mathrm{~mm}$ depth of field. Moreover, we will see in Sec. IV B that we can find a position in which both FZPs have the same resolution.

\section{B. Metrology on EQUINOX}

The EQUINOX laser facility ${ }^{11,12}$ features a $800 \mathrm{~nm}$ wavelength Ti:sapphire laser producing $0.3 \mathrm{~J}$ pulses, focused 


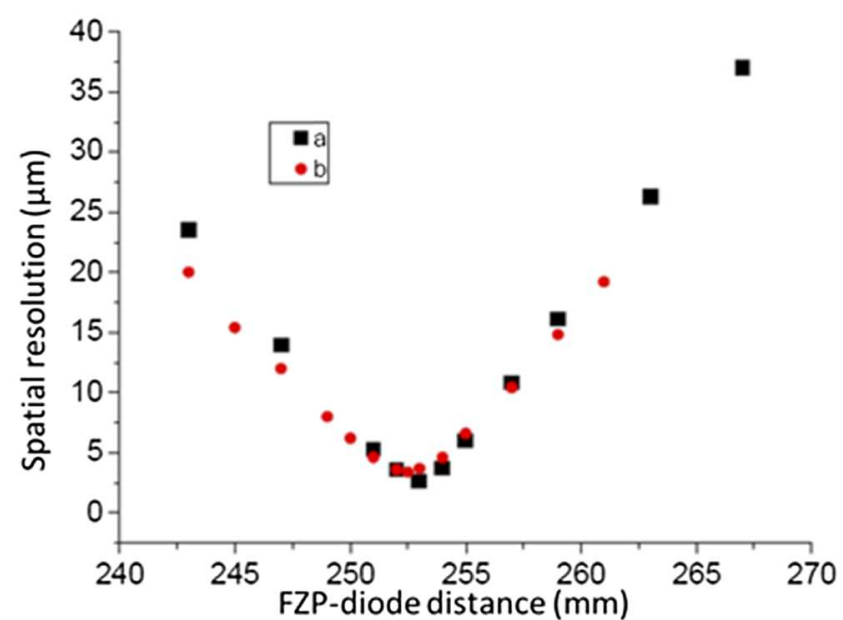

(a)

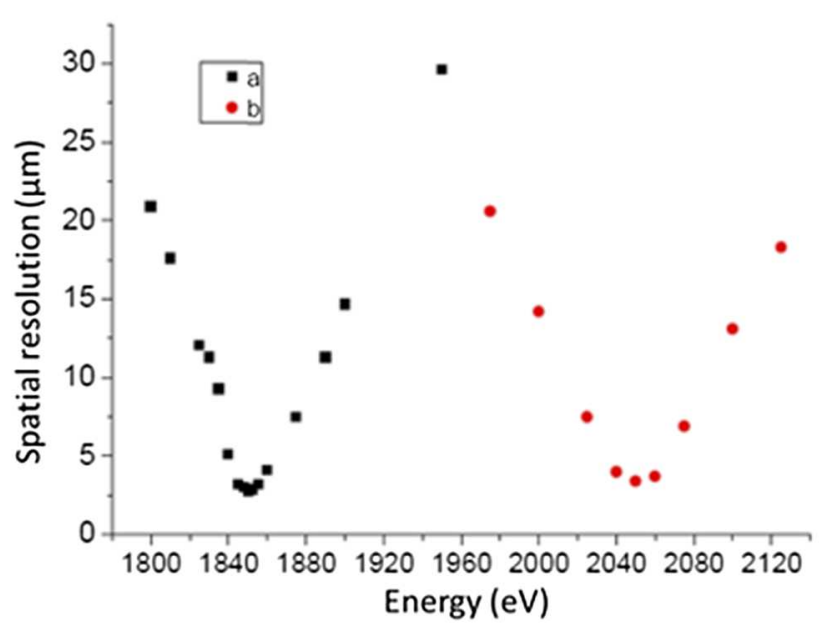

(b)

FIG. 2. Resolution measurements for both FZPs vs (a) FZP-diode distance for an energy of $1850 \mathrm{eV}$ for FZP $a$ and $2050 \mathrm{eV}$ for FZP $b$ and (b) energy for a FZP-diode distance of $253 \mathrm{~mm}$ for FZP $a$ and $252.5 \mathrm{~mm}$ for FZP $b$.

by an off-axis parabola. The laser pulse duration can be adjusted from 80 fs to $30 \mathrm{ps}$. As the focal spot diameter is about $50 \mu \mathrm{m}$, the irradiance on a flat solid target varies between $10^{14}$ and $10^{17} \mathrm{~W} / \mathrm{cm}^{2}$ and mostly produces X-rays in the $100 \mathrm{eV}-2 \mathrm{keV}$ range. The laser can operate at $10 \mathrm{~Hz}$ for signal accumulation.

Experiments at this facility measured the resolution by imaging a 500 mesh gold grid backlit with a thick Al target irradiated by the EQUINOX laser. Each image was accumulated over 100 shots.

We did not combine any spectral selection optics with our FZPs, meaning that the whole spectrum of our target is collected by our detector, resulting in a contrast and resolution loss giving us an upper limit for each FUHRIx 2 channel. In a previous paper, ${ }^{2}$ we showed that FZPs need their distances to be adjusted to the object position to match their calculated focal distance for a photon energy of $1630 \mathrm{eV}\left(\mathrm{Al} \mathrm{He}_{\alpha}\right)$. Considering the relation between the focal distance $\left(f_{\lambda}\right)$ and the wavelength $(\lambda)$,

$$
r_{1}^{2}=\lambda * f_{\lambda}
$$

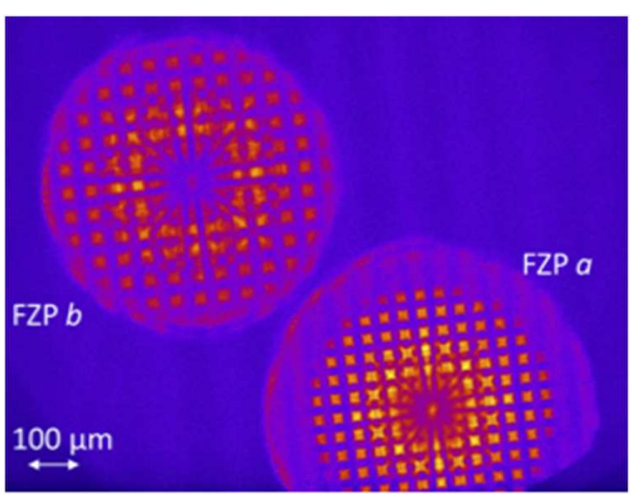

(a)

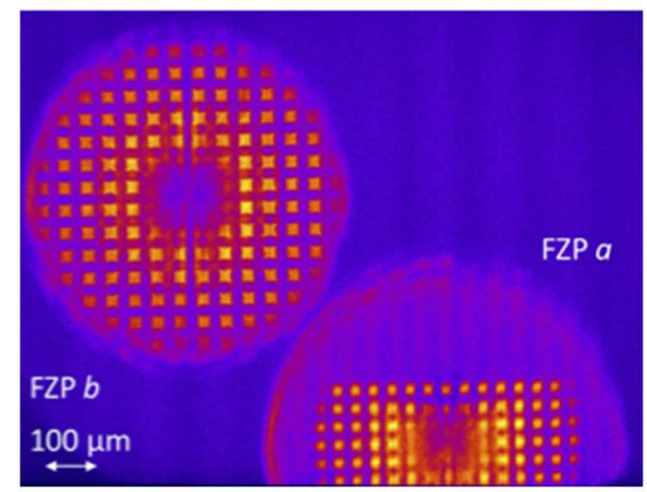

(b)

FIG. 3. Measured intensities (arbitrary units) of a gold grid imaged by the FZPs with a grid-FZP distance of (a) $241 \mathrm{~mm}$ and (b) $213 \mathrm{~mm}$.

where $r_{1}$ is the first zone radius, the distances are then calculated with the thin lens formula: $1 / f_{\lambda}=1 / p+1 / q$, with $p$ being the object-FZP distance and $q$ being the FZP-detector distance. In our case, we have a fixed $q$ distance of 2782 $\mathrm{mm}$, constrained by the facility dimensions. For the FZP $a$ (respectively FZP $b$ ), this corresponds to a $p$ distance of $239.2 \mathrm{~mm}$ (respectively $214 \mathrm{~mm}$ ). Figures 3(a) and 3(b) show the results of the grid imaging when the FZPs are placed close to these two values of $p$. The star shaped feature at the center of each FZP image is an artifact of our 0th order blocker.

The resolution was estimated by calculating the FWHM of the derivative of the signal across each edge of the whole grid. From the results presented in Table II, we observed that the best resolution at the optimum $p$ distance is very close for both FZPs $(\sim 4.7 \pm 0.5$ or $4.6 \pm 0.5 \mu \mathrm{m})$. These values are worse than the theoretical resolution of a FZP because of the $\mathrm{Al}$ large spectral bandwidth and the finite $\mathrm{X}$-ray source size produced by the laser pulse.

TABLE II. Resolution measurements in both directions for different $p$ distances.

\begin{tabular}{lccc}
\hline \hline & $p=241 \mathrm{~mm}$ & $p=213 \mathrm{~mm}$ & $p=223 \mathrm{~mm}$ \\
\hline FZP $a$ resolution $(\mu \mathrm{m})$ & $4.7 \pm 0.5$ & $9.0 \pm 0.4$ & $6.5 \pm 0.5$ \\
FZP b resolution $(\mu \mathrm{m})$ & $9.5 \pm 0.5$ & $4.6 \pm 0.4$ & $6.5 \pm 0.5$ \\
\hline \hline
\end{tabular}


We did a complementary resolution measurement by putting the grid between the optimal position of each FZP $(p=223 \mathrm{~mm})$. We found an identical resolution for both lenses of $6.5 \pm 0.5 \mu \mathrm{m}$.

\section{CONCLUSIONS}

FZPs present numerous advantages as imaging optics for ICF experiments. They have the potential to be designed with high $f$-number (190.5 and 211.1 for FZPs $a$ and $b$ respectively) that provides a high signal level despite their limited efficiency.

Moreover, high resolution in the micron range was demonstrated. We measured the spatial resolution of a bi-lens FZP that is going to be used as part of a high-resolution quasimonochromatic X-ray microscope for laser-plasma interaction experiments. We can estimate the resolution of each imaging channel by giving their upper and lower limits:

\section{$2.4 \mu \mathrm{m} \leq$ FUHRIx 2 channel $a$ resolution $\leq 5.2 \mu \mathrm{m}$}

$2.4 \mu \mathrm{m} \leq$ FUHRIx 2 channel $b$ resolution $\leq 5.1 \mu \mathrm{m}$.

We can also calculate an approximate value for the expected resolution when these FZPs are coupled to a CCD camera by using Eq. (2) and assuming that our CCD camera is identical to the one previously measured on an experiment $\left(r_{C C D}=2.3 \mu \mathrm{m}\right)$. This gives an approximate expected system resolution of $3.3 \mu \mathrm{m} \leq r_{d i a} \leq 5.7 \mu \mathrm{m}$. This will be verified in an upcoming experiment at LULI2000 laser facility (LULI, Ecole Polytechnique, France).

One of the currently overlooked uses of FZPs is as complementary spectroscopic diagnostics. Using an array of FZPs with different spectral selection not only would allow imaging of an object emitting in multiple atomic lines with a high resolution, but would also allow performing an analysis of a spatially resolved line ratio.

\section{ACKNOWLEDGMENTS}

The authors acknowledge Michael Krumrey, Levent Cibik, and Swenja Schreiber (both PTB) for their help during the experiments as well as Laboratoire de Photonique et de Nanostructures (LPN-CNRS, Marcoussis, France) lab team for their help in imaging FZPs with a scanning electron microscope.

${ }^{1}$ J. D. Lindl, Inertial Confinment Fusion (Springer-Verlag, New York, 1998).

${ }^{2}$ L. A. Pickworth et al., Rev. Sci. Instrum. 85, 11D611 (2014).

${ }^{3} \mathrm{Ph}$. Troussel, B. Meyer, R. Reverdin, B. Angelier, G. Lidove, P. Salvatore, and A. Richard, Rev. Sci. Instrum. 76, 63707 (2005).

${ }^{4}$ A. Do et al., Rev. Sci. Instrum. 88, 013701 (2017).

${ }^{5}$ See http://www.luli.polytechnique.fr/accueil/les-installations/luli2000 for more information about LULI2000 website.

${ }^{6}$ S. Gorelick, J. Vila-Comamala, V. A. Guzenko, R. Barrett, M. Salomé, and C. David, J. Synchrotron Radiat. 18(3), 442-446 (2011).

${ }^{7}$ M. Krumrey and G. Ulm, Nucl. Instrum. Methods Phys. Res., Sect. A 467-468, 1175-1178 (2001).

${ }^{8}$ D. Fuchs, M. Krumrey, P. Müller, F. Scholze, and G. Ulm, Rev. Sci. Instrum. 66, 2248-2250 (1995).

${ }^{9}$ A. V. Baez, J. Opt. Soc. Am. 51, 405-412 (1961).

${ }^{10}$ J. Vila-Comamala, S. Gorelick, E. Färm, C. M. Kewish, A. Diaz, R. Barrett, V. A. Guzenko, M. Ritala, and C. David, Opt. Express 19, 175-184 (2011).

${ }^{11}$ J. P. LeBreton et al., Rev. Sci. Instrum. 77, 10F530 (2006).

${ }^{12}$ Ch. Reverdin, M. Paurisse, T. Caillaud, P. Combis, A. Duval, D. Gontier, D. Husson, Ch. Rubbelynck, and C. Zuber, Rev. Sci. Instrum. 79, 10E932 (2008).

${ }^{13}$ A. Do et al., "Two-channel high-resolution quasi-monochromatic X-ray imager for $\mathrm{Al}$ and Ti plasma," Rev. Sci. Instrum. (submitted). 